\title{
Analytical solution of the associative mean spherical approximation for the ion-dipole model
}

\author{
M.F.Holovko, V.Kapko \\ Institute for Condensed Matter Physics, National Academy of Sciences of \\ Ukraine, 1 Svientsitskii St., UA-290011 Lviv-11, Ukraine
}

Received May 19, 1998

\begin{abstract}
A simple electrolyte in a polar solvent is modelled by a mixture of polar hard spheres and equal diameter charged hard spheres with the possibility of ionic dimerization. The analytical solution of the associative mean spherical approximation (AMSA) for this model is derived to its full extent. Explicit expressions for pair correlation functions and dielectric constant in terms of the AMSA are established. Some numerical calculations illustrate the role of ionic association.
\end{abstract}

Key words: association, ion-dipole model, mean spherical approximation

PACS: $05.20 .-y$

\section{Introduction}

The mean spherical approximation (MSA) is an analytical theory for electrolyte solutions in the ionic approach [1-3], as well as in the ion-molecular approach [412]. The solution of MSA defines screening potentials which play an important role in the construction of the optimized cluster expansion for free energy and correlation functions [13-15]. The MSA corresponds to the well-known DebyeHuckel (DH) theory [16] in the low coupling Mayer limit (small ionic concentrations and low nonideality of electrostatic interactions) The MSA is also asymptotically correct in the high coupling Onsager limit (high densities and strong nonideality of electrostatic interactions) [17], where, unlike the DH theory, it satisfies the exact Onsager bounds [18] for the Helmholtz free energy and the internal energy of the system.

However, the MSA (similar to the DH theory) is a linearized theory (in the strength of electrostatic interactions and in the inverse temperature) that fails at low temperature and does not capture the full second ionic virial coefficient or the full effect of ion pairing. Moreover, the MSA, as a usual integral equation theory, is derived from the Mayer density expansion and does not satisfy the high coupling 
low-density limits. For strongly associating liquids an infinite number of terms in the density expansion need to be included in order to obtain the correct low-density limit, while only a few terms of the activity expansion are enough for this purpose [18-19]. A simple and natural way to correct the theory of electrolyte solutions was proposed by Bjerrum [20] who developed the theory of an ionic association in conjunction with the DH theory. In general, an ionic association occurs due to strong Coulomb interactions or as a result of the chemical mechanism caused by a true chemical bond formation. However, the first realization of an ionic association was done at the thermodynamical level with the application of the original Bjerrum idea, which is exactly correct only for point ions [21].

Recently, a multidensity integral equation theory which correctly accounts for the effects of association in ionic systems has been proposed [20-23]. It is based on the multidensity formalism for associating fluids of Wertheim [36-37] and combines a description in terms of the activity and density expansions. The description of ionic associations in the multidensity formalism with the extension MSA began from the two-density version which was referred to in [26] as an associative MSA (AMSA). The analytical solution of the AMSA was found for an ionic dimerization model [26] and then for an arbitrary mixture of dimerizing ions [27], for an ionic restricted primitive model [26], for a shielding sticky ionic model [32], for a highly asymmetric ionic model [29] and then it was generalized for polymerizing ions [2930] and also for ionic fluids with an arbitrary multiple bonding [31]. The explicit expression for thermodynamical properties of a mixture of dimerizing ions was obtained with the application of the exponential approximation for the contact values of a non-associated part of pair distribution functions $g_{a b}^{00}$ which was used for the calculation of the degree of association [36-37]. Such an approximation yields the correct Bjerrum limit for very dilute solutions and the AMSA corresponds to the DH theory with the Bjerrum corection for an ionic association in the limit of point ions. Moreover, as we have recently shown [21], the results of the AMSA can be supplemented by the Ebeling-Grigo [38] choice of the degree of association which provide the capture of the full second ionic virial coefficient. As a result, the solution of the AMSA of associating ionic systems is asymptotically correct in the Onsager high coupling and high density limits, as well as in a high coupling total association and low density limits.

The application of the AMSA to the electrolyte solution has been focused up to this time only on the development of an ionic approach. The application of the AMSA to an ion-molecular model was done for any case. The simplest such model is an ion-dipole mixture which has been solved analytically in the MSA for a restricted case of equal sizes of ions and dipoles $[4-6,10]$ and also for a more general case [7-9, 12-14].

From this article we begin to generalize the solution of the MSA for an iondipole model for the AMSA case. In general, in ion-molecular systems, ion-molecular and molecular associations can exist, except for an ionic association. An ion-molecular association describes ionic solvation effects and a molecular association can be connected with $\mathrm{H}$-bonding effects. Important aspects of molecular 
systems with hydrogen bonds connected with the proton transfer were developed by Stasyuk and co-workers [39]. However, in this article for simplification we restrict the consideration of the ion-dipole model only to an ionic association. A more general ion-diple model with molecular and ion-molecular association will be considered elsewhere [41].

The article is organized as follows. In the second section an ion-dipole model with an ionic association is introduced and a general scheme of the analytical solution of the AMSA for this model is presented. In the third section explicit expressions for pair correlation functions and the dielectric constant are obtained for the AMSA solution. Some explicit numerical results, as well as some conclusions are given in the fourth section.

\section{The model and the general scheme of the AMSA solution}

The considered model consists of a three-component mixture of hard spheres with embedded point dipoles and equal size hard spheres with embedded point charges fulfilling the charge neutrality condition. The oppositely charged hard spheres may have associative interactions, which are unique for dimerizing particles. The potential model employed in our study can be presented in the form:

$$
U_{x y}\left(X_{1}, X_{2}\right)=U_{x y}^{H S}\left(r_{12}\right)+U_{x y}^{e l}\left(X_{1}, X_{2}\right)+\left(1-\delta_{x y}\right) U_{x y}^{d i m}\left(z_{12}\right),
$$

where $U_{x y}^{H S}\left(r_{12}\right)$ is the potential of the hard spheres of diameters $\sigma$,

$U_{x y}^{e l}\left(X_{1}, X_{2}\right)$ are the pairwise electrostatic interaction potentials, $X_{1}$ denotes set of coordinates of particle 1 and $\Omega_{1}$ is a set of the Euler angles necessary to define the orientation of the molecule, $r_{12}$ is the interparticle distance, $U_{x y}^{\operatorname{dim}}\left(z_{12}\right)$ is the associative potential which appears due to a sticky point site placed on the surface of each hard ion. Here $z_{12}$ is the distance between the sticky sites of two ions at a given centre-centre separation and orientation.

Our solution is based upon the Baxter-Wertheim factorization technique and follows the scheme developed earlier. The AMSA can be expressed in terms of the Wertheim-Ornstein-Zernike (WOZ) equation for mixtures

$$
\begin{gathered}
h_{x y}^{\alpha \beta}\left(X_{1}, X_{2}\right)=c_{x y}^{\alpha \beta}\left(X_{1}, X_{2}\right)+\sum_{z} \sum_{\gamma \delta} \int \mathrm{d} X_{3} h_{x z}^{\alpha \gamma}\left(X_{1}, X_{3}\right) \rho_{z}^{\gamma \delta} \\
c_{z y}^{\delta \beta}\left(X_{3}, X_{2}\right)
\end{gathered}
$$

together with AMSA closure relations

$$
\begin{aligned}
& h_{x y}^{\alpha \beta}\left(X_{1}, X_{2}\right)=-\delta_{\alpha 0} \delta_{\beta 0}, \quad r_{12}<1 \\
& c_{x y}^{\alpha \beta}\left(X_{1}, X_{2}\right)=-\delta_{\alpha 0} \delta_{\beta 0} * \beta_{T} U_{x y}^{e l}\left(X_{1}, X_{2}\right)+ \\
& \delta_{\alpha 1} \delta_{\beta 1}\left(1-\delta_{x y}\right) B g_{+-}^{00}\left(r_{12}\right) \delta(r-1+), r_{12} \geqslant 1,
\end{aligned}
$$

where we put $\sigma=1$ and consider $\sigma$ as a unit of length, $\beta_{T}=1 /\left(k_{b} T\right)$ is the Boltzmann thermal factor, $B$ is the strength parameter of the association between sticky points placed on the surface, $\delta(r)$ is the Dirac delta function. $h_{x y}^{\alpha \beta}\left(X_{1}, X_{2}\right)$ 
and $c_{x y}^{\alpha \beta}\left(X_{1}, X_{2}\right)$ are a pair correlation function and a direct correlation function, respectively, for species $\mathrm{x}$ and $\mathrm{y}$ in positions $\vec{r}_{1}$ and $\vec{r}_{2}$. For dipolar particles these functions will depend on the orientation. Thus, the notation $h_{x y}^{\alpha \beta}\left(X_{1}, X_{2}\right)$ includes the relative distance $r_{12}=\left|\vec{r}_{1}-\vec{r}_{2}\right|$, as well as possible orientations $\hat{s}_{1}$ and $\hat{s}_{2}$ of dipole moments, with $\hat{s}$ being a unit vector along the dipolar moment, $d X_{3}$ denotes an integration over the position $r_{3}$ and orientations $\hat{s}_{3}$ (with $\int d \hat{s}_{3}=\frac{1}{4 \pi} \int d \Omega=1$ ). The indices $\alpha, \beta, \gamma, \delta$ have the values 0,1 for ions and 0 for dipoles and indicate the degree of association of the corresponding particle. The case $\alpha=0$ corresponds to an unbonded particle, $\alpha=1$ - to the particle with a bonded site. For simplicity we will omit index " $\alpha$ " for dipoles.

$\rho_{x}$ is the density of the particle of type $\mathrm{x}$, which is separated into two densities $\rho_{z}=\rho_{z}^{0}+\rho_{z}^{1}$, where $\rho_{z}^{0}$ is the density of monomer particles, $\rho_{z}^{1}$ - the density of dimers; $\rho_{z}^{00}=\rho_{z}, \rho_{z}^{01}=\rho_{z}^{10}=\rho_{z}^{0}, \rho_{z}^{11}=0$. Owing to the pair potential (1) and to the condition of electroneutrality $\rho_{+}=\rho_{-}, \rho_{+}^{0}=\rho_{-}^{0}$. For dipoles $\rho_{d}=\rho_{d}^{0}$. The monomer and total ionic densities are related by a self-consistent relation [26]

$$
\rho_{i}=\rho_{i}^{0}+2 \pi\left(\rho_{i}^{0}\right)^{2} B g_{+-}^{00}(1+),
$$

where $\rho_{i}=\rho_{+}+\rho_{-}, \rho_{i}^{0}=\rho_{+}^{0}+\rho_{-}^{0}$.

All orientation-dependent functions are presented in the orientation-invariant form $[10,14,41]$

$$
f_{x y}^{\alpha \beta}\left(X_{12}\right)=\sum_{m n l} f_{x y}^{\alpha \beta}\left(\left.r_{12}\right|^{m n l}\right) \Phi^{m n l}\left(\Omega_{1}, \Omega_{2}, \Omega_{r_{12}}\right)
$$

where

$$
\begin{gathered}
\Phi^{m n l}\left(\Omega_{1}, \Omega_{2}, \Omega_{r_{12}}\right)=\sum_{\alpha \beta \lambda} l !\left(\begin{array}{c}
m n l \\
\alpha \beta \lambda
\end{array}\right) /\left(\begin{array}{c}
m n l \\
000
\end{array}\right) * \\
D_{0 \alpha}^{m}\left(\Omega_{1}\right) D_{0 \beta}^{n}\left(\Omega_{2}\right) D_{0 \lambda}^{l}\left(\Omega_{r_{12}}\right), \\
f_{x y}^{\alpha \beta}\left(\left.r_{12}\right|^{m n l}\right)=\frac{(2 m+1)(2 n+1)(2 l+1)}{l !}\left(\begin{array}{c}
m n l \\
000
\end{array}\right) \sum_{m n l}\left(\begin{array}{c}
m n l \\
\alpha \beta \lambda
\end{array}\right) * \\
\int \mathrm{d} \Omega_{1} \mathrm{~d} \Omega_{2} \mathrm{~d} \Omega_{r_{12}} D_{0 \alpha}^{* m}\left(\Omega_{1}\right) D_{0 \beta}^{* n}\left(\Omega_{2}\right) D_{0 \lambda}^{* l}\left(\Omega_{r_{12}}\right) f_{x y}^{\alpha \beta}\left(X_{12}\right) .
\end{gathered}
$$

Here the standard notation for the Wigner $3-j$ symbol is applied; $D_{0 \alpha}^{m}(\Omega)$ are generalized spherical functions. In our particular case this reduces to

$$
\begin{aligned}
& f_{i i}^{\alpha \beta}\left(X_{12}\right)=f_{i i}^{\alpha \beta}\left(\left.r\right|^{000}\right), \\
& f_{i d}^{\alpha}\left(X_{12}\right)=f_{i d}^{\alpha}\left(\left.r\right|^{000}\right)+f_{i d}^{\alpha}\left(\left.r\right|^{011}\right) \Phi^{011}\left(\Omega_{2}, \Omega_{r}\right), \\
& f_{d d}\left(X_{12}\right)=f_{d d}\left(\left.r\right|^{000}\right)+f_{d d}\left(\left.r\right|^{110}\right) \Phi^{110}\left(\Omega 1, \Omega_{2}\right)+ \\
& f_{d d}\left(\left.r\right|^{112}\right) \Phi^{112}\left(\Omega 1, \Omega_{2}, \Omega_{r}\right),
\end{aligned}
$$

where "i" and "d" denote ions and dipoles, respectively. 
We have

$$
\begin{aligned}
& \Phi^{011}\left(\Omega_{2}, \Omega_{r}\right)=\hat{r} \hat{s}_{2}, \\
& \Phi^{110}\left(\Omega_{1}, \Omega_{2}\right)=\hat{s}_{1} \hat{s}_{2}, \\
& \Phi^{112}\left(\Omega_{1}, \Omega_{2}, \Omega_{r}\right)=3\left(\hat{s}_{1} \hat{r}\right)\left(\hat{s}_{2} \hat{r}\right)-\left(\hat{s}_{1} \hat{s}_{2}\right),
\end{aligned}
$$

where $\hat{r}$ is a unit vector along $\vec{r}$ and $\hat{s}$ - along the dipolar moment.

The set of WOZ equations can be divided into two formally independent sets of equations by introducing the following linear combinations:

$$
\begin{aligned}
& h_{i i}^{(S) \alpha \beta}(r)=\frac{1}{2}\left[h_{++}^{\alpha \beta}\left(\left.r\right|^{000}\right)+h_{+-}^{\alpha \beta}\left(\left.r\right|^{000}\right)\right], \\
& h^{\alpha \beta}\left(\left.r\right|^{000}\right)=\frac{1}{2}\left[h_{++}^{\alpha \beta}\left(\left.r\right|^{000}\right)-h_{+-}^{\alpha \beta}\left(\left.r\right|^{000}\right)\right], \\
& h_{i d}^{(S) \alpha}(r)=\frac{1}{2}\left[h_{+d}^{\alpha}\left(\left.r\right|^{011}\right)+h_{-d}^{\alpha}\left(\left.r\right|^{011}\right)\right], \\
& h^{\alpha}\left(\left.r\right|^{011}\right)=\frac{1}{2}\left[h_{+d}^{\alpha}\left(\left.r\right|^{011}\right)-h_{-d}^{\alpha}\left(\left.r\right|^{011}\right)\right], \\
& h_{d d}^{(S)}(r)=h_{d d}\left(\left.r\right|^{000}\right), \\
& h\left(\left.r\right|^{110}\right)=h_{d d}\left(\left.r\right|^{110}\right), \\
& h\left(\left.r\right|^{112}\right)=h_{d d}\left(\left.r\right|^{112}\right) .
\end{aligned}
$$

Similar combinations we have for $c_{x y}^{\mu \nu}\left(\left.r\right|^{m n l}\right)$. We begin from the solution of a set of equations for the electrostatic part.

\subsection{Solution of a set of equations for the electrostatic part}

The closure relations (3) for the electrostatic part of the correlation functions can be written in the following form:

$$
\begin{gathered}
h^{\alpha \beta}\left(\left.r\right|^{m n l}\right)=0 \text { ifr }<1, \\
c^{\alpha \beta}\left(\left.r\right|^{000}\right)=-\delta_{\alpha 0} \delta_{\beta 0} \beta_{T} \frac{e^{2}}{r}-\frac{1}{2} \delta_{\alpha 1} \delta_{\beta 1} B g_{+-}^{00}(1+) \delta(r-1+), \\
c^{\alpha}\left(\left.r\right|^{011}\right)=\delta_{\alpha 0} \beta_{T} \frac{e p_{d}}{r^{2}}, \\
c^{\alpha}\left(\left.r\right|^{110}\right)=0 \text { if } r \geqslant 1, \\
c\left(\left.r\right|^{112}\right)=\beta_{T} \frac{p_{d}^{2}}{r^{3}},
\end{gathered}
$$

where e and $p_{d}$ are an ionic charge and a dipole moment, respectively.

The WOZ has to be solved in the Fourier space. Therefore, the three-dimensional Fourier transform of $h_{m n l}^{\alpha \beta}(r)$ (and $c_{m n l}^{\alpha \beta}(r)$ ) is a Hankel transform of the order $1:$

$$
h_{m n l}^{\alpha \beta}(k)=4 \pi i^{l} \int_{0}^{\infty} r^{2} \mathrm{~d} r j_{l}(k r) h_{m n l}^{\alpha \beta}(r),
$$

where $j_{l}(k r)$ is a spherical Bessel function of the order 1. As it was shown earlier for the MSA case [6-11], for the solution of the WOZ equation we can introduce 
the functions

$$
\begin{aligned}
J^{\alpha \beta}\left(\left.r\right|_{\lambda} ^{m n}\right)= & \frac{2 \pi\left[\rho_{m} \rho_{n}\right]^{\frac{1}{2}}}{[(2 m+1)(2 n+1)]^{\frac{1}{2}}} \sum_{l} l !\left(\begin{array}{c}
m n l \\
\lambda-\lambda 0
\end{array}\right) /\left(\begin{array}{c}
m n l \\
000
\end{array}\right) * \\
& \int_{|r|}^{\infty} r^{\prime} P_{l}\left(\frac{r}{r^{\prime}}\right) h^{\alpha \beta}\left(\left.r^{\prime}\right|^{m n l}\right) \mathrm{d} r^{\prime},
\end{aligned}
$$

where $0 \leqslant \lambda \leqslant \min (m, n), P_{l}(x)$ is the Legendre polynomial of the order 1 . The similar functions $S^{\alpha \beta}\left(\left.r\right|_{\lambda} ^{m n}\right)$ should be introduced as transformations of $c^{\alpha \beta}\left(\left.r\right|^{m n l}\right)$.

As a result, the WOZ equation divides into two different sets of equations in the Fourier space, which can be written as equations between $J_{11}^{\prime}(r)=J\left(\left.r\right|_{1} ^{11}\right)$ and $S_{11}^{\prime}(r)=S\left(\left.r\right|_{1} ^{11}\right)$ and a matrix equation with $\hat{J}(r)=J^{\alpha \beta}\left(\left.r\right|_{0} ^{m n}\right)$ and $\hat{S}(r)=$ $S^{\alpha \beta}\left(\left.r\right|_{0} ^{m n}\right)$

In this way the WOZ equation reduces to

$$
\hat{J}(k)-\hat{S}(k)=\hat{J}(k) \times \hat{\sigma} \times \hat{S}(k)
$$

together with

$$
J_{11}^{\prime}(k)-S_{11}^{\prime}(k)=-J_{11}^{\prime}(k) S_{11}^{\prime}(k),
$$

where $\hat{J}(k), \hat{S}(k), J^{\prime}(k)$ and $S^{\prime}(k)$ are one-dimensional Fourier transforms of the corresponding functions $\hat{J}(r), \hat{S}(r), J^{\prime}(r)$ and $S^{\prime}(r)$, the matrix

$$
\hat{\sigma}=\left[\begin{array}{ccc}
1 & x_{i} & 0 \\
x_{i} & 0 & 0 \\
0 & 0 & 1
\end{array}\right]
$$

and

$$
x_{i}=\rho_{i}^{0} / \rho_{i} .
$$

From (10) and (12), the AMSA closure condition can be written as

$$
\begin{gathered}
\hat{J}(r)=\left[\begin{array}{ccc}
b_{0}^{00} & b_{0}^{01} & 0 \\
b_{0}^{10} & b_{0}^{11} & 0 \\
0 & 0 & b_{0}^{\prime}
\end{array}\right]+\left[\begin{array}{ccc}
0 & 0 & b_{1}^{0} \\
0 & 0 & b_{1}^{1} \\
-b_{1}^{0} & -b_{1}^{1} & 0
\end{array}\right] r+\left[\begin{array}{ccc}
0 & 0 & 0 \\
0 & 0 & 0 \\
0 & 0 & b_{2}
\end{array}\right] r^{2} \quad \text { if } r<1 . \\
J_{11}^{\prime}=b_{0}^{\prime \prime}-\frac{1}{2} b_{2} r^{2} \text { if } r<1,
\end{gathered}
$$

where from equation (12),

$$
\begin{aligned}
& b_{0}^{\alpha \beta}=2 \pi \rho_{i} \int_{1}^{\infty} r h^{\alpha \beta}\left(\left.r\right|^{000}\right) \mathrm{d} r, \\
& b_{1}^{\alpha}=2 \pi \sqrt{\rho_{i} \rho_{d} / 3} \int_{1}^{\infty} h^{\alpha}\left(\left.r\right|^{011}\right) \mathrm{d} r,
\end{aligned}
$$




$$
\begin{gathered}
b_{2}=2 \pi \rho_{d} \int_{1}^{\infty} h\left(\left.r\right|^{112}\right) / r \mathrm{~d} r \\
b_{0}^{\prime}=\frac{2 \pi \rho_{d}}{3} \int_{1}^{\infty} r\left[h\left(\left.r\right|^{110}\right)-h\left(\left.r\right|^{112}\right)\right] \mathrm{d} r, \\
b_{0}^{\prime \prime}=\frac{2 \pi \rho_{d}}{3} \int_{1}^{\infty} r\left[h\left(\left.r\right|^{110}\right)+\frac{1}{2} h\left(\left.r\right|^{112}\right)\right] \mathrm{d} r . \\
\hat{S}(r)=\left[\begin{array}{ccc}
-\frac{d_{0}^{2} e^{-\mu r}}{2 \mu} & 0 & \frac{d_{0} d_{2}}{2} e^{-\mu r} \\
0 & -z \Theta(1-r) & 0 \\
-\frac{d_{0} d_{2}}{2} e^{-\mu r} & 0 & 0
\end{array}\right] \quad \text { if } r \geqslant 1 \\
S_{11}^{\prime}(r)=0 \quad \text { if } r \geqslant 1,
\end{gathered}
$$

where $d_{0}^{2}=4 \pi \beta_{T} e^{2} \rho_{i}, d_{2}^{2}=\frac{4 \pi}{3} \beta_{T} p_{d}^{2} \rho_{d}$,

$$
z=\pi B \rho_{i} g_{+-}^{00}(1+),
$$

$\mu$ is a factor introduced to avoid divergences. In what follows the limit $\mu \rightarrow 0$ is considered. Here it must be noted that the decoupled equation (14) together with the closure relations (18) and (21) are nothing but a transformed hard spheres Percus-Yevick equation in which the hard sphere density can be expressed as

$$
\rho=-b_{2} / 2 \pi \text {. }
$$

Equation (13) together with the closure relations (17) and (20) are suitably solved by means of the Baxter-Wertheim factorization method. This factorization is given by

$$
\hat{1}-\hat{\sigma} \times \hat{S}(k)=\hat{\sigma} \times \hat{Q}(k) \times \hat{\sigma} \times \hat{Q}^{T}(-k),
$$

where

$$
\hat{\sigma} \times \hat{Q}(k)=\hat{1}-\int_{0}^{\infty} \hat{\sigma} \times \hat{Q}(r) e^{i k r} \mathrm{~d} r .
$$

$\hat{1}$ is a unity $3 \times 3$ matrix, and the symbol $\mathrm{T}$ denotes a transposed matrix. $\hat{Q}(r)$ will be a simple matrix function of the form:

$$
\hat{Q}(r)=\hat{Q}^{0}-\hat{A} e^{-\mu r},
$$

where $\hat{A}$ is a constant matrix and $\hat{Q}^{0}(r)$ is a short-ranged matrix function

$$
\hat{Q}^{0}(r)=0 \text { if } r>1 \text {. }
$$

Explicit relations between $\hat{Q}(r)$ and the quantities $\hat{J}(r)$ and $\hat{S}(r)$ can be obtained in $r$ space.

$$
\hat{S}(r)=\hat{Q}(r)-\int_{0}^{\infty} \mathrm{d} t \hat{Q}(r+t) \times \hat{\sigma} \times \hat{Q}^{T}(t)
$$


and

$$
\hat{J}(r)=\hat{Q}(r)+\int_{0}^{\infty} \mathrm{d} t \hat{J}(r-t) \times \hat{\sigma} \times \hat{Q}(t) .
$$

By analysing equation (28) and taking into account the closure (20), we obtain:

$$
\hat{Q}^{0}(1+)-\hat{Q}^{0}(1-)=\hat{Z}
$$

where

$$
\hat{A}=\left[\begin{array}{ccc}
a_{1} & a_{2} & a_{3} \\
0 & 0 & 0 \\
0 & 0 & 0
\end{array}\right]
$$

and

$$
\hat{Z}=\left[\begin{array}{lll}
0 & 0 & 0 \\
0 & z & 0 \\
0 & 0 & 0
\end{array}\right] \text {. }
$$

The solution of the AMSA reduces to the calculation of the matrix factor function $\hat{Q}(r)$. Using some algebra we find its elements, which can be presented in the form:

$$
\begin{aligned}
\hat{Q}^{0}(r) & =\hat{Q}^{(1)}(r-1)+\hat{Q}^{(2)}\left(r^{2}-1\right)-\hat{Z}, \\
Q_{11}^{(1)} & =\frac{1}{\Delta}\left(D_{1} a_{1}-\frac{1}{2}\left(b_{1}^{0}\right)^{2}\right), \\
Q_{12}^{(1)} & =\frac{1}{\Delta}\left(D_{1} a_{2}-\frac{1}{2} b_{1}^{0}\left(b_{1}^{1}+x_{i} b_{1}^{0} z\right)\right), \\
Q_{13}^{(1)} & =\frac{1}{\Delta}\left(D_{1} a_{3}+b_{1}^{0}\left(1+\frac{1}{3} b_{2}\right)\right), \\
Q_{21}^{(1)} & =\frac{1}{\Delta}\left(D_{2} a_{1}-\frac{1}{2} b_{1}^{0} b_{1}^{1}\right), \\
Q_{22}^{(1)} & =\frac{1}{\Delta}\left(D_{2} a_{2}-\frac{1}{2} b_{1}^{1}\left(b_{1}^{1}+x_{i} b_{1}^{0} z\right)\right), \\
Q_{23}^{(1)} & =\frac{1}{\Delta}\left(D_{2} a_{3}+b_{1}^{1}\left(1+\frac{1}{3} b_{2}\right)\right), \\
Q_{31}^{(1)} & =\frac{1}{\Delta}\left(D_{3} a_{1}-b_{1}^{0}\left(1-\frac{2}{3} b_{2}\right)\right), \\
Q_{32}^{(1)} & =\frac{1}{\Delta}\left(D_{3} a_{2}-\left(b_{1}^{1}+x_{i} b_{1}^{0} z\right)\left(1-\frac{2}{3} b_{2}\right)\right), \\
Q_{33}^{(1)} & =\frac{1}{\Delta}\left(D_{3} a_{3}-\frac{b_{1}^{0}}{2}\left(b_{1}^{0}+2 x_{i} b_{1}^{1}\right)-\frac{\left(b_{2}\right)^{2}}{2}\right), \\
Q_{11}^{(2)} & =Q_{12}^{(2)}=Q_{13}^{(2)}=0, \\
Q_{21}^{(2)} & =Q_{22}^{(2)}=Q_{23}^{(2)}=0, \\
Q_{31}^{(2)} & =\frac{1}{\Delta}\left(D_{4} a_{1}-\frac{b_{1}^{0} b_{2}}{2}\right), \\
Q_{32}^{(2)} & =\frac{1}{\Delta}\left(D_{4} a_{2}-\frac{b_{2}}{2}\left(b_{1}^{1}+x_{i} b_{1}^{0} z\right)\right), \\
Q_{33}^{(2)} & =\frac{1}{\Delta}\left(D_{4} a_{3}+b_{2}\left(1+\frac{1}{3} b_{2}\right)\right),,
\end{aligned}
$$


where

$$
\begin{aligned}
& D_{1}=J_{0}\left(1-\frac{1}{6} b_{2}\right)^{2}+\frac{x_{i} b_{1}^{0}}{4}\left(J_{0} b_{1}^{1}-J_{1} b_{1}^{0}\right)- \\
& \frac{b_{1}^{0}}{3}\left(b_{1}^{0}+x_{i} b_{1}^{1}\right)\left(1-\frac{b_{2}}{24}\right), \\
& D_{2}=J_{1}\left(1-\frac{1}{6} b_{2}\right)^{2}-\frac{1}{4}\left(b_{1}^{0}+x_{i} b_{1}^{1}\right)\left(J_{0} b_{1}^{1}-J_{1} b_{1}^{0}\right)- \\
& \frac{b_{1}^{1}}{3}\left(b_{1}^{0}+x_{i} b_{1}^{1}\right)\left(1-\frac{b_{2}}{24}\right), \\
& D_{3}=-\frac{1}{2}\left[J_{0}\left(b_{1}^{0}+x_{i} b_{1}^{1}\right)+J_{1} x_{i} b_{1}^{0}\right]\left(1-\frac{2}{3} b_{2}\right)+\frac{b_{1}^{0}}{6}\left(b_{1}^{0}+x_{i} b_{1}^{1}\right) * \\
& \left(b_{1}^{0}+2 x_{i} b_{1}^{1}\right)+\frac{b_{2}}{4}\left(b_{1}^{0}+x_{i} b_{1}^{1}\right), \\
& D_{4}=-\frac{1}{4}\left[J_{0}\left(b_{1}^{0}+x_{i} b_{1}^{1}\right)+J_{1} x_{i} b_{1}^{0}\right] b_{2}-\frac{b_{1}^{0}}{8}\left(b_{1}^{0}+x_{i} b_{1}^{1}\right) * \\
& \left(b_{1}^{0}+2 x_{i} b_{1}^{1}\right)-\frac{1}{2}\left(b_{1}^{0}+x_{i} b_{1}^{1}\right)\left(1+\frac{b_{2}}{3}\right), \\
& \Delta=\left(1-\frac{b_{2}}{6}\right)^{2}+\frac{b_{1}^{0}}{4}\left(b_{1}^{0}+2 x_{i} b_{1}^{1}\right), \\
& J_{0}=b_{0}^{00}+x_{i} b_{0}^{01}, \\
& J_{1}=b_{0}^{10}+x_{i} b_{0}^{11} .
\end{aligned}
$$

The contact value of the function $h^{00}\left(1+\left.\right|^{000}\right)$ follows from (29) and (12)

$$
\begin{aligned}
& h^{00}\left(1+\left.\right|^{000}\right)=\frac{1}{2 \pi \Delta}\left[\left[\left(\left(1-\frac{1}{6} b_{2}\right)^{2}+\frac{1}{4} x_{i} b_{1}^{0} b_{1}^{1}\right) J_{0}-\right.\right. \\
& \left.\left.\frac{1}{4} x_{i}\left(b_{1}^{0}\right)^{2} J_{1}-\frac{1}{3} b_{1}^{0}\left(b_{1}^{0}+x_{i} b_{1}^{1}\right)\left(1-\frac{1}{24} b_{2}\right)\right] * a_{1}-\frac{1}{2}\left(b_{1}^{0}\right)^{2}\right] .
\end{aligned}
$$

\subsection{Solution of a set of equations for $\hat{h}^{(S)}(r)$}

A set of equations for a non-electrostatic part of correlation functions $\hat{h}^{(S)}(r)$ can be written in the Fourier space.

$$
\hat{h}^{(S)}(k)=\hat{c}^{(S)}(k)+\hat{c}^{(S)}(k) \times \hat{\rho} \times \hat{h}^{(S)}(k),
$$

where $\hat{h}^{(S)}(k), \hat{c}^{(S)}(k)$ and $\hat{\rho}$ are matrices defined as follows:

$$
\begin{gathered}
\hat{h}^{(S)}(k)=\left[\begin{array}{ccc}
h_{i i}^{(S) 00}(k) & h_{i i}^{(S) 01}(k) & h_{i d}^{(S) 0}(k) \\
h_{i i}^{(S) 10}(k) & h_{i i}^{(S) 11}(k) & h_{i d}^{(S) 1}(k) \\
h_{d i}^{(S) 0}(k) & h_{d i}^{(S) 1}(k) & h_{d d}^{(S)}(k)
\end{array}\right], \\
\hat{\rho}=\left[\begin{array}{ccc}
\rho_{i} & 0 & 0 \\
0 & \rho_{i} & 0 \\
0 & 0 & \rho_{d}
\end{array}\right] \times \hat{\sigma},
\end{gathered}
$$


$\hat{h}_{x y}^{(S) \alpha \beta}(k), \hat{c}_{x y}^{(S) \alpha \beta}(k)$ denote the Fourier transforms of the correlation functions $\hat{h}_{x y}^{(S) \alpha \beta}(r)$ and $\hat{c}_{x y}^{(S) \alpha \beta}(r)$, respectively. A set equations (37) with closure conditions

$$
\begin{array}{r}
h_{x y}^{(S) \alpha \beta}(r)=-\delta_{\alpha 0} \delta_{\beta 0} \text { if } r \\
c_{x y}^{(S) \alpha \beta}(r)=\frac{1}{2} \delta_{x y} \delta_{\alpha 1} \delta_{\beta 1} B g_{+-}^{00}(1+) \delta(r-1+) \text { if } r \geqslant 1
\end{array}
$$

has a form similar to the mixture of hard spheres and dimerizing hard spheres. This is a particular case of mixture of dimerizing hard spheres which was solved analytically in [42]. The only exception is that the closure (40) includes function $\frac{1}{2} g_{+-}^{00}(r)$ instead of $g_{x y}^{(S)} 00(r)$. This difference does not, however, change the general scheme of the analytical solution in both cases. The solution can be presented in the form:

$$
\begin{array}{r}
\hat{Q}^{(S)}(r)=\hat{Q}^{S(1)}(r-1)+\hat{Q}^{S(2)}\left(r^{2}-1\right)+\hat{Z}, \\
Q_{11}^{S(1)}=Q_{13}^{S(1)}=Q_{31}^{S(1)}=Q_{33}^{S(1)}=-\frac{3 \pi \eta}{(1-\eta)^{2}}, \\
Q_{11}^{S(2)}=Q_{13}^{S(2)}=Q_{31}^{S(2)}=Q_{33}^{S(2)}=\frac{\pi(1+2 \eta)}{(1-\eta)^{2}}, \\
Q_{12}^{S(1)}=Q_{32}^{S(1)}=-Q_{12}^{S(2)}=-Q_{32}^{S(2)}=-\frac{\pi z \rho_{i}^{0}}{1-\eta}, \\
Q_{21}^{S(1)}=Q_{22}^{S(1)}=Q_{23}^{S(1)}=Q_{21}^{S(2)}=Q_{22}^{S(2)}=Q_{23}^{S(2)}=0,
\end{array}
$$

where $\eta=\frac{\pi}{6}\left(\rho_{i}+\rho_{d}\right)$. The contact value for $g_{i i}^{(S) 00}(r)$ :

$$
g_{i i}^{(S) 00}(1+)=1+h_{i i}^{(S) 00}(1+)=\frac{1+\eta / 2}{(1-\eta)^{2}} .
$$

Finally, using the results of the previous section we have the expression for $g_{+-}^{00}(1+)$

$$
g_{+-}^{00}(1+)=\frac{1+\eta / 2}{(1-\eta)^{2}}-h^{00}\left(1+\left.\right|^{000}\right),
$$

where $h^{00}\left(1+\left.\right|^{000}\right)$ is given in $(36)$.

\subsection{A set of nonlinear equations}

After a substitution of equation (26) in the k-space into (24) the matrix equation is obtained

$$
\begin{aligned}
& \hat{1}-\hat{\sigma} \times \hat{S}(k)=\hat{\sigma} \times \hat{Q}^{0}(k) \times \hat{\sigma} * \hat{Q}^{0 T}(-k)+\hat{\sigma} \times \hat{Q}^{0}(k) \times \hat{\sigma} \times \\
& \hat{A}^{T} \frac{1}{i k}-\hat{\sigma} \times \hat{A} \times \hat{\sigma} \times \hat{Q}^{0 T}(-k) \frac{1}{i k}+\hat{\sigma} \times \hat{A} \times \hat{\sigma} \times \hat{A}^{T} \frac{1}{k^{2}} .
\end{aligned}
$$

From the asymptotic behaviour of $\hat{S}(r)$, contained in equations (20), we can obtain in [10] (for small $\mathrm{k}$ and $\mu=0$ ):

$$
\hat{1}-\hat{\sigma} * \hat{S}(k)=\left(\begin{array}{ccc}
d_{0}^{2} \frac{1}{k^{2}} & -S_{12}(k)-\alpha S_{22}(k) & d_{0} d_{2} \frac{1}{i k} \\
\alpha d_{0}^{2} \frac{1}{k^{2}} & 1-\alpha S_{12}(k) & \alpha d_{0} d_{2} \frac{1}{i k} \\
-d_{0} d_{2} \frac{1}{i k} & -S_{32}(k) & d_{2}^{2}+y_{1}^{2}
\end{array}\right),
$$


where $y_{1}=\left(1-\frac{1}{6} b_{2}\right) /\left(1+\frac{1}{12} b_{2}\right)^{2}$.

Comparing equations (45) and (46), when k equals zero, the following set of equations is obtained:

$$
\begin{aligned}
& a_{1}^{2}+2 \alpha a_{1} a_{2}+a_{3}^{2}=d_{0}^{2}, \\
& K_{31}\left(a_{1}+\alpha a_{2}\right)+K_{32} * \alpha a_{1}+\left(K_{33}-1\right) * a_{3}=d_{0} d_{2}, \\
& K_{31}^{2}+2 \alpha K_{31} * K_{32}+\left(K_{33}-1\right)^{2}=d_{2}^{2}+\frac{\left(1-\frac{1}{6} b_{2}\right)^{2}}{\left(1+\frac{1}{12} b_{2}\right)^{4}}, \\
& a_{1}\left(K_{21}+\alpha K_{22}\right)+a_{2}\left(\alpha K_{21}-1\right)+a_{3} K_{23}=0,
\end{aligned}
$$

where

$$
K_{m n}=\int_{0}^{1} Q_{m n}^{0}(r) \mathrm{d} r=-\frac{1}{2} Q_{m n}^{S(1)}-\frac{2}{3} Q_{m n}^{S(2)} .
$$

Taking into account equations (33), (28) and (26) we can write

$$
\begin{aligned}
& \lim _{r \rightarrow 0+} \frac{d}{d r} \hat{S}(r)=\hat{Q}^{(1)}+\frac{1}{2} \hat{A} \times \hat{\sigma} \times \hat{A}^{T}-\hat{Z} \times \hat{\sigma} \times \hat{A}^{T}+\hat{Q}^{(1)} \times \hat{\sigma} \times \\
& \hat{A}^{T}+\hat{Q}^{(2)} \times \hat{\sigma} \times \hat{A}^{T}-\hat{Q}^{(1)} \times \hat{\sigma} \times \hat{Z}-\hat{Q}^{(2)} \times \hat{\sigma} \times \hat{Z}+\frac{1}{2} \hat{Q}^{(1)} \times \\
& \hat{\sigma} \times \hat{Q}^{(1) T}+\frac{1}{2} \hat{Q}^{(2)} \times \hat{\sigma} \times \hat{Q}^{(2) T}+\frac{1}{3} \hat{Q}^{(2)} \times \hat{\sigma} \times \hat{Q}^{(1) T}+ \\
& \frac{2}{3} \hat{Q}^{(1)} \times \hat{\sigma} \times \hat{Q}^{(2) T} .
\end{aligned}
$$

Similarly to ionic systems with an association [26] we have

$$
\lim _{r \rightarrow 0+} \frac{d S_{m n}(r)}{d r}=0 \text { for } S_{11}, S_{12}, S_{21}, S_{22}, S_{33} .
$$

We additionally have five equations. We can rewrite equation (14) as

$$
x_{i}+2 x_{i}^{2} \rho_{i} z=1
$$

Equations (47-50), (53), (54) and (22) are a set of eleven equations for ten variables $a_{1}, a_{2}, a_{3}, J_{0}, J_{1}, b_{1}^{0}, b_{1}^{1}, b_{2}, x_{i}, z$. In order to solve this system of equations one can apply the numerical methods (a standard Newton-Raphson technique, for example). It is necessary to note, that among the set of equations (53) for $S_{12}$ and $S_{22}$ and equation (50) only two are independent. We cannot prove this analytically due to the complexity of the obtained system. But numerical calculations confirm the identity of these sets.

\section{The pair distribution functions}

In this section we intend to develop explicit expressions to compute the correlation functions. As usual, we have

$$
j_{m n}(r)=\frac{\mathrm{d} J_{m n}(r)}{\mathrm{d} r} .
$$


From equation (29) for $r>1$

$$
\begin{aligned}
j_{m n}(r)= & \sum_{l k} \int_{0}^{1} j_{m l}(r-t) \sigma_{l k} Q_{k n}^{0}(t) \mathrm{d} t-\sum_{l k} \int_{1}^{r} j_{m l}(t) \sigma_{l k} A_{k n} \mathrm{~d} t+ \\
& \delta_{m 2} \rho_{i}^{0} z Q_{1 n}^{0}(r-1) \Theta(2-r)-c_{m} a_{n}
\end{aligned}
$$

where $c_{1}=J_{0}, c_{2}=J_{1}+\rho_{i}^{0} z, c_{3}=-\left(b_{1}^{0}+\alpha b_{1}^{1}\right)$.

To obtain $j_{m n}(r)$ for $r>1$, we may use the iterative scheme of Perram [43]. Knowing these functions it is possible to find the pair distribution function according to the scheme:

$$
\begin{aligned}
& H_{00}(r)=-\frac{1}{2 \pi r}\left(j_{11}(r)+\alpha\left(j_{12}(r)+j_{21}(r)+\alpha j_{22}(r)\right)\right), \\
& H_{01}(r)=-\frac{1}{2 \pi r}\left(j_{13}(r)+\alpha j_{23}(r)\right) \text {, } \\
& H_{10}(r)=-H_{01}(r) \text {, } \\
& H_{11}(r)=-\frac{1}{2 \pi r} j_{33}(r) \text {, } \\
& H_{11}^{\prime}(r)=\rho h_{p y}(r) b y \rho=-\frac{b_{2}}{2 \pi}, \\
& H^{000}(r)=H_{00}(r) \\
& H^{011}(r)=\sqrt{3} H_{01}(r), \\
& H^{110}(r)=H_{11}(r)+2 H_{11}^{\prime}(r) \text {, } \\
& H^{112}(r)=H_{11}(r)-H_{11}^{\prime}(r) \text {, } \\
& h^{000}(r)=H^{000}(r) / \rho_{i}, \\
& h^{110}(r)=H^{110}(r) / \rho_{d} \text {, } \\
& h^{011}(r)=\left(H^{011}(r)-\frac{1}{r^{2}} \int_{0}^{r} H^{011}(R) R \mathrm{~d} R\right) / \sqrt{\rho_{i} \rho_{d}}, \\
& h^{112}(r)=\left(H^{112}(r)-\frac{3}{r^{3}} \int_{0}^{r} H^{112}(R) R^{2} \mathrm{~d} R\right) / \rho_{d} .
\end{aligned}
$$

The latter are connected with the functions (9) by the relations:

$$
\begin{aligned}
& h^{000}(r)=h^{00}\left(\left.r\right|^{000}\right)+x_{i}\left(h^{01}\left(\left.r\right|^{000}\right)+h^{10}\left(\left.r\right|^{000}\right)+x_{i} h^{11}\left(\left.r\right|^{000}\right)\right), \\
& h^{011}(r)=h^{0}\left(\left.r\right|^{011}\right)+x_{i} h^{1}\left(\left.r\right|^{011}\right), \\
& h^{110}(r)=h\left(\left.r\right|^{110}\right), \\
& h^{112}(r)=h\left(\left.r\right|^{112}\right) .
\end{aligned}
$$


From (9) and (8) we can define the total pair distribution functions

$$
\begin{array}{r}
g_{++}(r)=g_{--}(r)=1+h_{i i}^{(S)}(r)-h_{000}(r), \\
g_{+-}(r)=1+h_{i i}^{(S)}(r)-h_{000}(r), \\
g_{+d}\left(r, \theta_{2}\right)=1+h_{i d}^{(S)}(r)+h_{011}(r) \cos \left(\theta_{2}\right), \\
g_{d d}\left(r, \theta_{1}, \theta_{2}\right)=1+h_{d d}^{(S)}(r)+h_{110}(r) \cos \left(\theta_{12}\right), \\
+h_{112}(r)\left(3 \cos \left(\theta_{1}\right) \cos \left(\theta_{2}\right)-\cos \left(\theta_{12}\right)\right) .
\end{array}
$$

Using the Aldeman expression [44] the dielectric constant can be written in the form:

$$
\epsilon=1+d_{2}^{2} \frac{\left(1+\frac{1}{12} b_{2}\right)^{4}}{\left(1-\frac{1}{6} b_{2}\right)^{2}} .
$$

\section{Discussion of the numerical results}

In this section we shall consider some AMSA numerical results of interest for electrolytes. The influence of ionic dimerization on the screening potentials $h^{000}(r)$, $h^{011}(r), h^{110}(r)$ and $h^{112}(r)$ is represented in figure 1. In this figure and the following
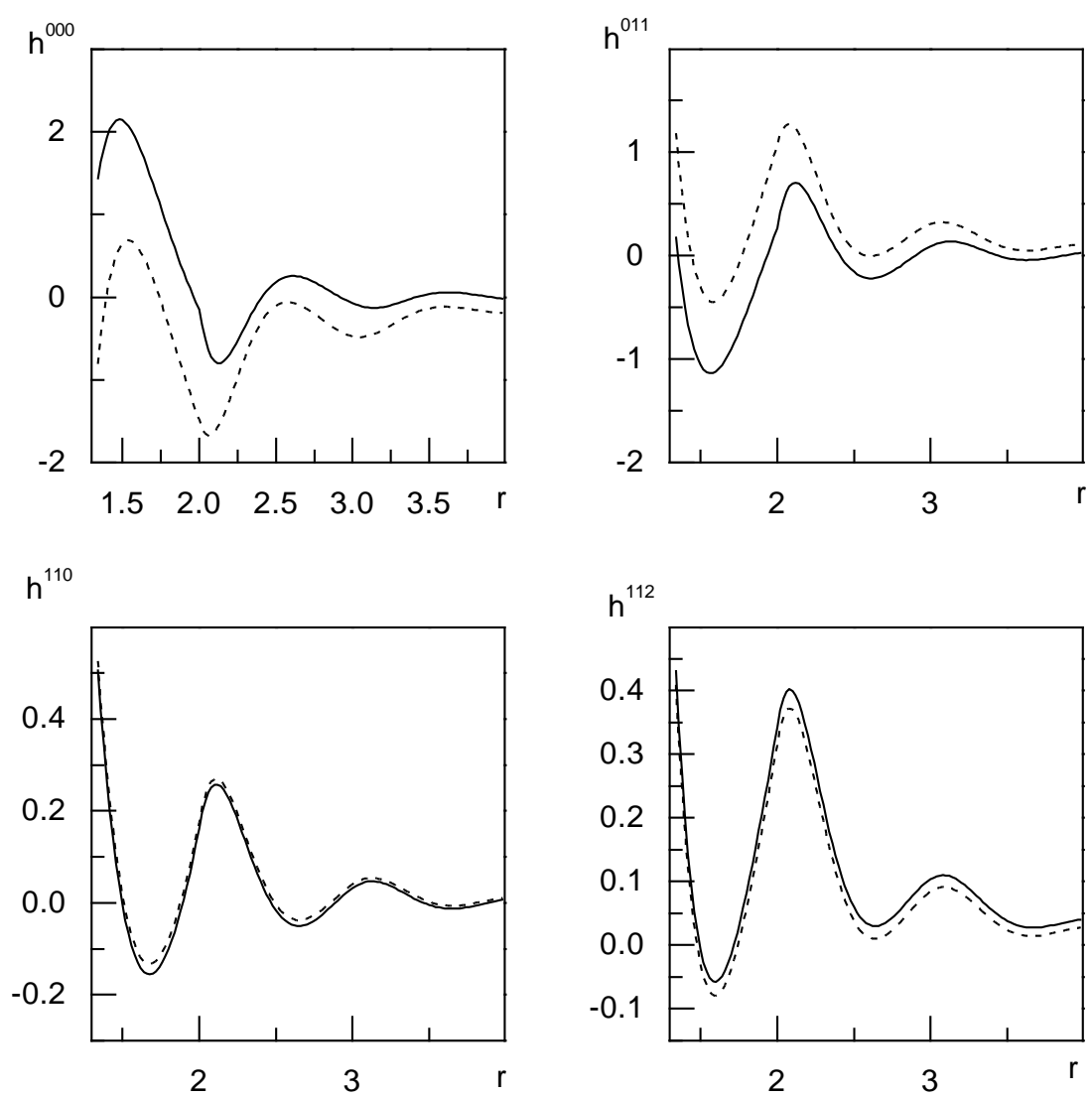

Figure 1. The electrostatic part of the correlation functions of the ion-dipole system with an ionic dimerization: - - - - B=0; $\mathrm{B}=1000$. 
ones we have used a set of dimensionless parameters to describe the model, namely:

$$
\beta_{i}=\frac{\beta_{T} e^{2}}{\sigma}, \beta_{d}=\frac{\beta_{T} p_{d}^{2}}{\sigma^{3}}, \rho^{*}=\frac{N_{i}+N_{d}}{V} \sigma^{3}, \quad \text { and } \quad c_{i}=\frac{N_{i}}{N_{i}+N_{d}} .
$$

We put $\rho^{*}=0.43975, \beta_{i}=2982.0, \beta_{d}=38.851$, which corresponds to aqueous electrolyte solutions of singly charged ions with the ionic size $\sigma=2.76 \AA$ at normal temperature and pressure. The data are represented for ionic concentration $c_{i}=$ $10^{-2}$ and for two values of the association parameter $B_{a s}$ :

1) for non-associative case $B_{a s}=0$ (dotted line);

2) for $B_{a s}=10^{3}$ which, in accordance with (4), corresponds to the monomer fraction $\rho_{i}^{0} / \rho_{i}=0.04$ (solid line). The ionic curves exhibit a well-known minimum at $\mathrm{r}=2[6,11,14]$ which shows the stability of positive ion-solvent molecule - negative ion triplets.

$h^{011}(r)$ describes the formation of an ionic solvation shell. The ionic dimerization leads to a more structural form of the functions $h^{000}(r), h^{011}(r)$ and $h^{112}(r)$, but not of $h^{110}(r)$. A more structured form of the screening potential is in agreement with the numerical solution of nonlinear integral equations for an ion-dipole model without an association. It means that association essentially improves the results of the usual MSA. An essential role of an assocation for ionic distribution functions is demonstrated in figure 2. We should note that due to an association, $g_{++}(r)$ includes a nonregular term $\frac{1}{4 \pi} \frac{\rho_{i}-\rho_{i}^{0}}{\rho_{i}} \delta(r-1)$ which is not presented in figure 2.
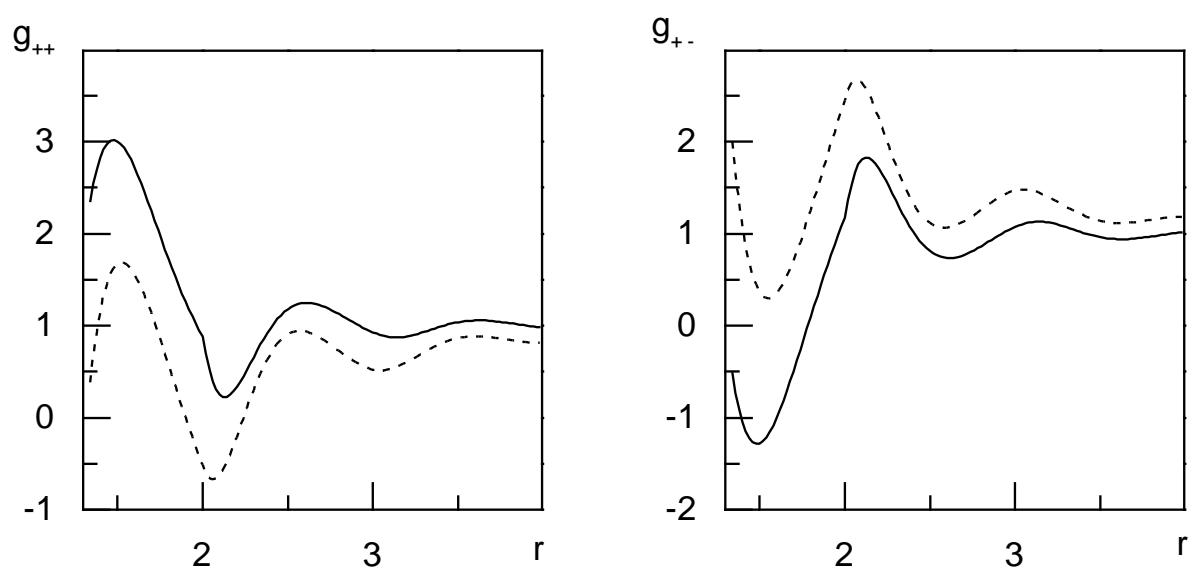

Figure 2. The ionic pair distribution functions: - - - $\mathrm{B}=0 ; \quad-\mathrm{B}=1000$.

The dependence of the fraction of undimerized ions $x_{i}=\frac{\rho_{i}^{0}}{\rho_{i}}$ as a function of ionic concentration is presented in figure 3. Similarly to the pure ionic model with the increasing ionic concentration, the process of ionic dimerization becomes more intensive. The above result is consistent with the Le Chatelier principle, since the process of olimerization can be treated as an exothermic reaction.

Finally, the dependence of the dielectric constant on the ionic concentration is represented in figure 4. As in the MSA, the dielectric constant decreases, but 
due the ionic dimerization this decrease is slowler compared with a non-associative case.

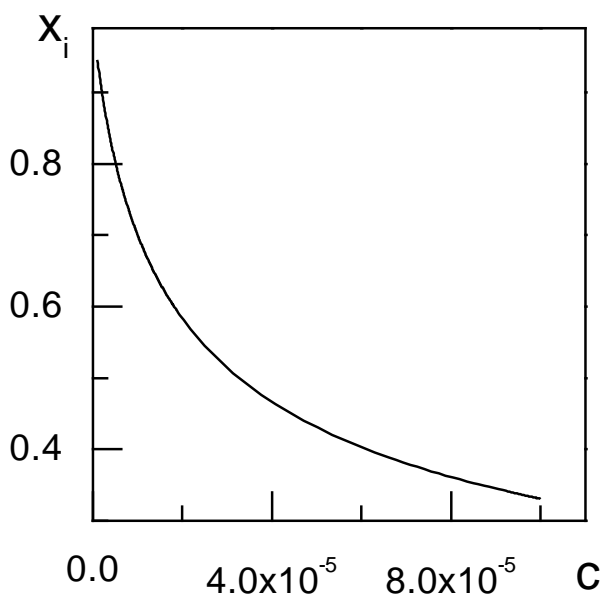

Figure 3. The fraction of undimerized ions vs an ionic concentration.

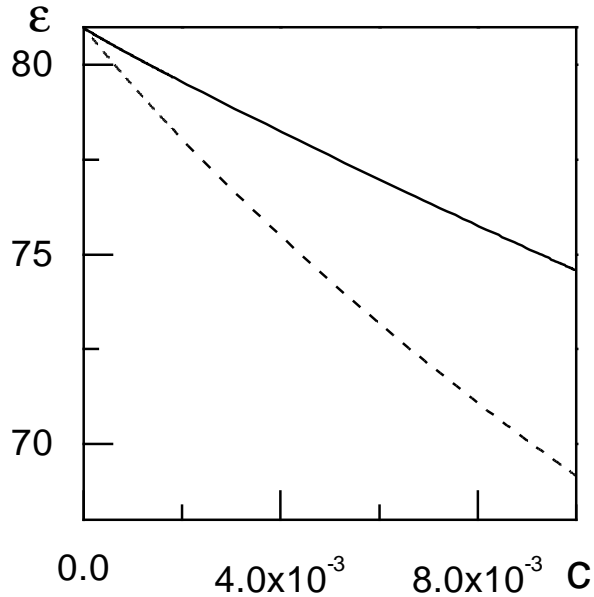

Figure 4. The dielectric constant as a function of the ionic concentration: - - . - $\mathrm{B}=0$; $\mathrm{B}=1000$.

\section{Acknowledgements}

This work was supported in part by a grant from the Fundamental Research Fund Program of Ukraine (2.4/174) and by the joint INTAS-Ukraine Call'95 grant (INTAS-UA95-133).

\section{References}

1. Waisman E. and Lebowitz J.L. // J. Chem. Phys., 1970, vol. 52, p. 430, 1972, vol. 56, p. 3086.

2. Blum L. // Mol. Phys., 1975, vol. 30, p. 1529.

3. Blum L. and Hoye J.S. // J. Phys. Chem., 1977, vol. 81, p. 1311

4. Blum L. // Chem. Phys. Lett., 1974, vol. 26, p. 200; J. Chem. Phys., 1974, vol. 61, p. 2129.

5. Vericat F., Blum L. // J. Stat. Phys., 1980, vol. 22, p. 593.

6. Holovko M.F. The statistical theory of mixed ion-molecular systems. Doctoral thesis. // Institute for Theoretical Physics, Kiev, 1980

7. Blum L., Wei D.Q. // J. Chem. Phys., 1987, vol. 87, p. 555

8. Wei D.Q., Blum L. // J. Chem. Phys., 1987, vol. 87, p. 2999.

9. Holovko M.F., Protsykevich I.A. // Chem. Phys. Lett., 1987, vol. 142, p. 463; J. Stat. Phys., 1989, vol. 54, p. 707.

10. Hoye J.S., Lomba E. // J. Chem. Phys, 1988, vol. 88, p. 5790

11. Hoye J.S., Lomba E., Stell G. // J. Chem. Phys, 1988, vol. 89, p. 7642

12. Protsykevych I.A. // Cond. Matt. Phys., 1993, vol. 2, p. 68. 
13. Fawcett W.R., Blum L. // Cond. Matt. Phys., 1993, vol. 2, p. 76.

14. Yukhnovsky I.R., Holovko M.F. The statistical theory of classical equilibrium systems. // Naukova dumka, Kiev, 1980.

15. Holovko M.F., Yukhnovsky I.R. In "Chemical Physics of Solvation", Elsevier, Amsterdam, 1985, vol. A, p. 207-262.

16. Holovko M.F., Sovyak E.M. // Cond. Matt. Phys., 1995, vol. 6, p. 49.

17. Rosenfeld Y., Blum L. // J. Phys. Chem., 1985, vol. 89, p. 5149; J. Chem. Phys., 1986, vol. 85, p. 1556.

18. Onsager L. // J. Phys. Chem., 1939, vol. 63, p. 189.

19. Debye P., Huckel E. // Phys. Z, 1923, vol. 24, p. 185.

20. Bjerrum N., Dan K. Vidensk. Felsk. Math. Fys. Medd., 1926, vol. 7, p. 9.

21. Ebeling W., Kraeft W.D., Kremp D. Theory of bound states and ionization equilibrium in plasmas and solids. // Akademic Verlag, Berlin, 1976

22. Kalyuzhnyi Yu.V., Holovko M.F., Haymet A.D.J. // J. Chem. Phys., 1991, vol. 95, p. 9151.

23. Barthel J., Holovko M.F., Kalyuzhnyi Yu.V., Krienke H., Protsykevitch I.A., Wittmann H. // J. Mol. Liq., 1988, (in press).

24. Kalyuzhnyi Yu.V., Vlachy V. // Chem. Phys. Lett., 1993, vol. 215, p. 518.

25. Kalyuzhnyi Yu., Vlachy V., Holovko M.F., Stell G. // J. Chem. Phys., 1995, vol. 102., p. 5770 .

26. Holovko M.F., Kalyuzhnyi Yu.V. // Mol. Phys., 1991, vol. 73, p. 1145.

27. Kalyuzhnyi Yu.V., Holovko M.F. // 1993, vol. 80, p. 1165.

28. Blum L., Bernard O. // J. Stat. Phys., 1995, vol. 79, p. 569.

29. Kalyuzhnyi Yu.V., Blum L., Holovko M.F., Protsykevytch I.A. // Physica A, 1997, vol. 236, p. 85.

30. Protsykevytch I.A., Kalyuzhnyi Yu.V., Holovko M.F., Blum L. // J. Mol. Liquids, 1997, vol. 1, p. 73-74.

31. Blum L., Holovko M.F., Protsykevytch I.A. // J. Stat. Phys., 1996, vol. 84, p. 191.

32. Duda Yu.Ya., Kalyuzhnyi Yu.V., Holovko M.F. // J. Chem. Phys., 1996, vol. 104, p. 1081.

33. Kalyuzhnyi Yu.V., Stell G. // Chem. Phys. Lett., 1995, vol. 157, p. 240.

34. Bernard O., Blum L. // J. Chem. Phys., 1996, vol. 104, p. 4746.

35. Kalyuzhnyi Yu.V., Holovko M.F. // J. Chem. Phys., 1998, vol. 108, p. 3709.

36. Wertheim M.S. // J. Stat. Phys., 1984, vol. 35, p. 19-35.

37. Wertheim M.S. // J. Stat. Phys., 1986, vol. 42, p. 459-477.

38. Ebeling W., Grigo M., // Ann. Phys., Leipzig, 1980, vol. 37, p. 21.

39. Stasyuk I.V., Ivankiv O.L., Pavlenko N.I. // J. Phys. Studies, 1997, vol. 1, p. 418.

40. Stasyuk I.V., Ivankiv O.L. // Mod. Phys. Lett., 1992, vol. B6, p. 85.

41. Holovko M.F., Kapko V. //J. Mol. Liq., 1998, (in press).

42. Kalyuzhnyi Yu.V., Holovko M.F. //J. Chem. Lett., 1993, vol. 215, p. 1.

43. Perram J.W. // Mol. Phys., 1975, vol. 30, p. 1505.

44. Adelman S.A. // J. Chem. Phys., 1976, vol. 64, p. 724. 


\title{
Аналітичний розв'язок асоціативного середньо-сферичного наближення для іонно-дипольної моделі
}

\author{
М.Ф.Головко, В.І.Капко
}

Інститут фізики конденсованих систем НАН України, 290011 м. Львів, вул. Свєнціцького, 1

Отримано 19 травня 1998 р.

Простий електроліт моделюється в полярному розчиннику сумішшю полярних твердих сфер і однакового розміру заряджених твердих сфер із можливою іонною димеризацією. Подано аналітичний розв'язок асоціативного середньо-сферичного наближення для цієї моделі (ACCH). Приводяться точні вирази для парних кореляційних функцій і діелектричної константи в термінах АCCH. Роль іонної асоціативності ілюструється числовими результатами.

Ключові слова: асоціативність, іонно-дипольна модель, середньо-сферичне наближення

PACS: 05.20. $-y$ 
